\title{
Influence of Intramuscular Dexamethasone on Labor Duration in primigravidas
}

Fatma Ahmed AboRomia

Azza Fouad Mohammed El-Adham

Lecturer of Obstetric and Gynecological Nursing

Faculty of Nursing, Tanta University

\begin{abstract}
The influence of intramuscular (IM) administration of dexamethasone on labor duration in primigravidas was assessed. An observational prospective case control design was used. Data collected from two Maternity and Child Healthcare Centers, over three months using a pre designed assessment tool conducted by the researchers.. The study sample was selected by purposive random sample technique. The sample consisted of 172 primigravidas women who had favorable cervix with a bishop score of 7 or greater, pregnancy duration of at least 37 weeks, a first trimester ultrasound, and a vertex presentation of a singleton fetus. Primigravidas with maternal systemic diseases, pre mature rupture of membranes (PROM), and vaginal bleeding were excluded. The sample was divided into two equal groups. The experimental group (86 primigravidas, 43 from each center) received an intramuscular injection of $8 \mathrm{mg}(2 \mathrm{ml})$ of dexamethasone, and the control group ( 86 primigravidas, 43 from each center) received a placebo of IM injection of $2 \mathrm{ml}$ of saline. Data was analyzed using SPSS Version 17. Data results revealed that women received (IM) injection of dexamethasone had significantly shorter duration of the second stage of labor compared to those received IM injection of $2 \mathrm{ml}$ of saline. However, no statistically significant differences were found between the studied groups regarding durations of the active / first and third stages of labor. The study recommended that intramuscular injection of dexamethasone is very important to shorten the length of the second stage of labor especially for primigravidas women to prevent unwanted complications.
\end{abstract}

\section{Introduction}

Although administrating corticosteroids is a suggested method to shorten labor duration, the role of these agents in the process of labor in women is not well understood. $^{(1)}$ Several animal studies have shown the importance of corticosteroid secretion by the fetal adrenal glands on the beginning of labor. ${ }^{(2)}$ The findings of these studies have led to the hypothesis that corticosteroids also has an effect on 
the duration of labor in primigravidas women. Corticotrophin-releasing hormone $(\mathrm{CRH})$ has been identified in various organ systems, including the female reproductive system. It is the principal regulator of the hypothalamic-pituitary-adrenal axis. Circulating placental corticotrophin releasing hormone $(\mathrm{CRH})$ is responsible for the physiologic hypercortisolism of the latter half of pregnancy and plays a role in the onset of labor. ${ }^{(3,4,5)}$

Administration of dexamethasone to pregnant women is now common practice if delivery before 34 weeks is expected. Dexamethasone reduces the incidence of respiratory distress syndrome in the newborn, enhances the efficacy of neonatal surfactant therapy, and reduces the associated risk of intravascular haemorrhage, and neonatal death. Conversely, some concerns exist about adverse maternal effects of corticosteroid. The study revealed the effect of dexamethasone on human chorionic gonadotrophin (HCG) concentrations and suggested a direct inhibitory effect on placental hormone synthesis or secretion. Recommendations included the need for further studies to define the mechanism of action of dexamethasone on placental HCG production. ${ }^{(6)}$
In this double-blind, randomized, placebocontrolled study, the effect of preoperative administration of dexamethasone on postoperative vomiting and pain in women undergoing general anesthesia for major gynecological surgery was evaluated. Dexamethasone was effective in reducing the overall incidence of vomiting from, and the influence of dexamethasone on postoperative pain was minimal. ${ }^{(7)}$ Dexamethasone is a corticosteroid with strong antiinflammatory and prolonged effective strong antiemetic in patients receiving cancer chemotherapy. It reduces the incidence of postoperative vomiting and surgery-related side effects such as delayed wound healing and increased incidence of wound infection. The cautious use of dexamethasone in surgical patients is recommended. Dexamethasone was administered orally, as well as intravenously (IV) as a post-operative antiemitic. Studies revealed that for antiemesis, the minimum effective dose of dexamethasone without discernible side effects is $2.5 \mathrm{mg}$. ${ }^{(8)}$

Dexamethasone is corticosteroids used in the management of preterm premature rupture of membranes (PPROM) to enhance fetal maturation and prevent obstetrical complication associated with high neonatal morbidity and mortality. 
Dexamethasone accelerates maturation of fetal lungs, decrease number of neonates with respiratory distress syndrome and improves survival in preterm delivered neonates. Optimal gestational age for use of dexamethasone therapy is 31 to 34 weeks of gestation. Thus, the prenatal administration of corticosteroids had great valuable effect in conditions of PPROM. $(9,10,11,12)$

Additionally, dexamethasone and lidocaine $1.5 \%$ solution are used in axillary brachial plexus block as they prolong the duration of sensory and motor blockade. The optimal dose of dexamethasone to be used for prolonged brachial plexus block as well as the mechanism of this effect is not clear. ${ }^{(13)}$ Dexamethasone is used in block prolongation of local anaesthetics. Dexamethasone prolonged the action of ropivacaine more than that of bupivacaine, the combined effect of dexamethasone and either drug produced nearly the same 22 hour of analgesia. Large studies will be necessary to demonstrate the safety of dexamethasone for perineural use. ${ }^{(14)}$

Medication used in women during labor and delivery, such as intramuscular injection of dexamethasone is implemented by obstetrics and gynecological nurses. They should understand the benefits and limitations of these medications. Obstetrics and gynecological nurses can contribute in the enhancement or alteration of the health conditions involving the mother, the fetus, and the newborn, because the scope of professional nursing practice is developed and expanded to include and allow nurses to implement a lot of clinical tasks and techniques that were not employed in the past. Obstetrics and gynecological nurses are key health care providers for childbearing women. They are significant personnel and have important roles in the prevention and control of morbidity and mortality risks of childbearing mothers, such ad prolonged exhausting labor and its subsequent complications. ${ }^{(15)}$

Many studies show the probable effects of corticosteroids on the labor process. Corticosteroids have been administered intravenously, intramuscularly, and by extra-amniotic infusion in various clinical trials. $^{(1,2,3,4)}$ Consequently, it is very important for obstetrics and gynecological nurses to find out the influence of intramuscular injection of dexamethasone on labor duration in primigravidas.

\section{Aim of the study}

The aim of the study was to determine the influence of intramuscular

(IM) 
administration of dexamethasone on duration of labor in primigravidas women.

\section{Materials and Method:}

\section{Materials}

\section{Study design}

An experimental prospective case control design to determine the influence of intramuscular (IM) administration of dexamethasone on duration of labor in primigravidas women.

\section{Setting}

The study was conducted at Segar and Kohafa Maternity and Child Healthcare Centers at Tanta, Egypt.

\section{Sample}

The study sample consisted of 172 primigravidas women. They were selected by purposive sample random technique and divided into two equal groups: the experimental and control group (86 primigravidas, 43 from each center), The sample size needed for each group can be determined by calculation or estimation of the eta-squared $\left(\eta^{2}\right)$, or based on information from relevant studies or a pilot study. Sample size ratios differ according to the number of groups in the study. Thus, for the current study, the approximate sample size necessary to achieve a level of power of $70 \%$ for $\alpha=.05$ as a function of estimated population values of eta-squared is (86) primigravidas in each of the two groups of the study. Consequently, there would be a $30 \%$ chance (power $=.70$ ) of finding non-significant results, even if the null hypothesis were false. ${ }^{(17)}$ Subjects were selected according to the following inclusion criteria: being primigravidas, having favorable cervix with a bishop score of 7 or greater, pregnancy duration of at least 37 weeks according to a reliable date for the last menstrual period and a first trimester ultrasound examination, vertex presentation and singleton fetus. The subjects' exclusion criteria comprised maternal systemic diseases, pre mature rupture of membranes (PROM), and vaginal bleeding.

\section{Tool of the study}

A pre designed assessment tool was developed by the researchers and used to collect the necessary data about the study sample. It included sociodemographic and clinical data, such as age in years, body mass index $\left(\mathrm{BME}=\right.$ weight $/$ height $\left.^{2}\right)$, gestational age, and the bishop score (assessment and calculation of the parameter/score of position, consistency, effacement, and dilatation of the cervix, and the foetal station).

\section{Methods}

Permission to carry out the study was obtained from the directors and the responsible specialists of the selected 
settings after explanation of the aim of the study. Informed, oral consent was obtained from all primigravidas women who were invited and agreed to participate after explanation of the aim of the study. They were informed of their voluntary to participate and the right to refuse and of the respect of their confidentiality. Before embarking in the study a pilot study was carried out on 8 primigravidas women (4 from each center) to determine the applicability of the study. These primigravidas were excluded from the actual data of the study. A total number of 172 primigravidas women were randomly selected and divided into two equal groups: the experimental group (86 primigravidas, 43 from each center), and the control group (86 primigravidas, 43 from each center). Gynecological procedures were enrolled in this study. The field work was carried out by the researchers within duration of three months. Once labor began the experimental group received an intramuscular injection of $8 \mathrm{mg}(2 \mathrm{ml})$ of dexamethasone, and the control group received a placebo IM $2 \mathrm{ml}$ of saline.

\section{Data collection}

Sociodemographic and clinical data (age in years, body mass index (BME), gestational age, and the bishop score) were assessed by the researchers using the pre designed assessment tool. The Body Mass Index (BMI) of the primigravidas was estimated by measuring their weight in kilograms and dividing it by the square of their height in meters. Bishop's Score calculation included assessment and calculation of the parameter/score of position, consistency, effacement, and dilatation of the cervix, and the foetal station. Parameter/score of cervix position ranged from (0 to 2$)$ which means posterior, intermediate, and anterior respectively). The anterior position is better aligned with the uterus, and therefore there is an increased likelihood of spontaneous delivery. Parameter/score of cervix consistency ranged from (0 to2) which means firm, intermediate, and soft respectively. When the cervix is less rigid, it allows for easier dilatation of the cervix at term as in multigravida woman. Parameter/score of cervical effacement are (0-30\%, 31-50\%, 51-80\%, and >80\%) which ranged from (0-3). It measures the stretch already present in the cervix and is affected by individual variations and surgery. Parameter/score of cervical dilatation are $(0 \mathrm{~cm}, 1-2 \mathrm{~cm}, 3-4 \mathrm{~cm}$, and $<5 \mathrm{~cm})$ which ranged from $(0-3)$. It is the diameter of the stretched cervix and it complements with effacement and is the most important indicator of progression of the first stage of labor. Parameters of fetal station are $(-3,-2,-1.0,+1$, and +2$)$ which ranged from (0-4). It describes the position of the fetal head in relation to the ischial spines (negative numbers indicates that the 
head is further inside above the ischial spines). It is palpated deeply inside the posterior vagina $(8-10 \mathrm{~cm})$ as a bony protrusion. The total Bishop Score is 14. A total Bishop Score for childbearing woman is considered favorable if the score is ( 7 or <7). Additionally, all women were subjected to full history taking, abdominal examination, assessment of fundal level and auscultation of fetal heart sounds.

\section{Statistical analysis}

tatistical analysis of the collected data was computed using SPSS versions 17 under the platform of Microsoft Windows 7. Continuous data were expressed in the form of mean $\pm \mathrm{SD}$, while categorical data were expressed in the form of count and percent. Comparison of continuous data was performed utilizing student $\mathrm{t}$ test, while categorical data were done using

Chi-square test. Relation between variables was investigated using Pearson's correlation coefficient. ( $\mathrm{P}$ value less than 0.05) was considered statistically significant.

\section{Results}

Table (1) shows that Intramuscular (IM) injection of $8 \mathrm{mg}(2 \mathrm{ml})$ dexamethasone was applied to the experimental group and a placebo IM injection of $2 \mathrm{ml}$ saline was applied to the control group once labor begins. Data were analyzed on age in years, body mass index (BME), gestational age, and the bishop score. Comparison between the studied groups There were no statistically significant differences between the two groups.

Table (2) shows that the Comparison between the 2 groups regarding duration of the various stages of labor using Student $t$ test had shown that women of the experimental group who received an intramuscular (IM) injection of $8 \mathrm{mg}(2 \mathrm{ml})$ dexamethasone had significantly shorter duration of the second stage of labor, the data results were statistically significant at $0.05 \%(\mathrm{P}=0.001)$ than the control group who received a placebo $\mathrm{IM} 2 \mathrm{ml}$ of saline once Labor begins. However, no statistically significant differences were found between the studied groups regarding durations of the active/first and third stages of labor

Table (3) illustrated the Correlation between durations of the three stages of labor and the basic data (age in years,body mass index (BME), gestational age, and the bishop score) revealed that a statistically significant relation at $0.05 \%$ existed between the subjects' BMI, gestational age, Bishop Score, and the duration of the first stage of labor $(\mathrm{P}=0.005,0.01$, and .0 .03$)$ respectively On the other hand, there was a statistically significant relation between the subjects' age and the duration of the second stage of labor $(\mathrm{P}=0.01)$. 
Table-1 Comparison between the basic findings in the studied groups

\begin{tabular}{|l|c|r|c|c|}
\hline \multicolumn{1}{|c|}{$\begin{array}{c}\text { Socio- } \\
\text { demographic and } \\
\text { clinical variables }\end{array}$} & $\begin{array}{r}\text { Dexamethasone } \\
\text { group }(\mathbf{n = 8 6})\end{array}$ & $\begin{array}{r}\text { Control group } \\
(\mathbf{n = 8 6})\end{array}$ & \multicolumn{2}{|c|}{ Student t test } \\
\cline { 4 - 5 } & $18.6 \pm 1.4$ & $18.8 \pm 1.7$ & -0.67 & 0.5 \\
\hline Age $($ Years) & $27.0 \pm 3.3$ & $26.4 \pm 2.7$ & 0.93 & 0.35 \\
\hline BMI $\left(\mathbf{K g} / \mathbf{m}^{\mathbf{2}}\right)$ & $38.2 \pm 1.0$ & $38.5 \pm 1.1$ & -1.1 & 0.27 \\
\hline Gestational age & $7.7 \pm 0.7$ & $7.9 \pm 0.8$ & -1.2 & 0.2 \\
\hline Bishop score & & & & \\
\hline
\end{tabular}

Table-2 Comparison between the studied groups regarding duration of the various stages of labor

\begin{tabular}{|l|c|c|c|c|}
\hline Duration of labor & Dexamethasone & \multirow{2}{*}{$\begin{array}{c}\text { Control group } \\
\text { group }(\mathbf{n = 8 6})\end{array}$} & \multicolumn{2}{|c|}{ Student t test } \\
\cline { 4 - 5 } & & & $\mathbf{T}=\mathbf{8 6})$ & $\mathbf{P}$ \\
\hline $\mathbf{1}^{\text {st }}$ stage duration & $3.6 \pm 0.7$ & $3.8 \pm 0.8$ & 0.81 & 0.15 \\
\hline $\mathbf{2}^{\text {nd }}$ stage duration & $17.9 \pm 3.5$ & $22.4 \pm 8.6$ & -3.4 & $0.001^{*}$ \\
\hline $\mathbf{3}^{\text {rd }}$ stage duration & $6.9 \pm 1.9$ & $7.0 \pm 1.8$ & 0.53 & 0.71 \\
\hline
\end{tabular}

Table-3 Correlation between $1^{\text {st }} \cdot 2^{\text {nd }}$, and $3^{\text {rd }}$ stages of labor duration and the basic data

\begin{tabular}{|l|c|c|c|c|c|c|}
\hline \begin{tabular}{c} 
Socio-demographic \\
\multirow{2}{*}{$\begin{array}{c}\text { and clinical } \\
\text { variables }\end{array}$}
\end{tabular} & \multicolumn{2}{c|}{$\begin{array}{c}\mathbf{1}^{\text {st }} \text { stage } \\
\text { duration }\end{array}$} & \multicolumn{2}{c|}{$\begin{array}{c}\mathbf{2}^{\text {nd }} \text { stage } \\
\text { duration }\end{array}$} & \multicolumn{2}{c|}{$\begin{array}{c}3^{\text {rd }} \text { stage } \\
\text { duration }\end{array}$} \\
\cline { 2 - 7 } Age (Years) & $\mathbf{r}$ & $\mathbf{P}$ & $\mathbf{R}$ & $\mathbf{P}$ & $\mathbf{r}$ & $\mathbf{p}$ \\
\hline BMI (Kg/m $\mathbf{2})$ & 0.11 & 0.12 & 0.19 & $0.01^{*}$ & 0.05 & 0.32 \\
\hline Gestational age & 0.19 & $0.005^{*}$ & 0.14 & 0.09 & -0.11 & 0.07 \\
\hline Bishop score & 0.17 & $0.01^{*}$ & 0.1 & 0.11 & 0.07 & 0.83 \\
\hline
\end{tabular}

\section{Discussion}

In the present study, comparison between the studied groups regarding duration of the various stages of labor had shown that the dexamethasone group had significantly shorter duration of the second stage of labor when compared with the control group. These are in line with the conclusions of Barkai et al. ${ }^{(1,2,17)}$ who found that inducing labor by means of an extra-amniotic infusion of corticosteroids through an intracervical Foley balloon catheter reduced the time between induction of labor and delivery. Also,
Kashanian et al. ${ }^{(18)}$ reported on the extraamniotic infusion of a saline solution mixed with dexamethasone through a Foley catheter whose balloon was filled with $15 \mathrm{~mL}$ of water, and concluded that the procedure could shorten the duration of labor without significant maternal or fetal risk. Thus, the present study provides high-level evidence of direct relevance for clinical practice, because the use of dexamethasone on labor of primigravidas will reduce the risk of prolonged labor and 
delivery and prevent the consequent complications.

Ziaei et al. ${ }^{(19)}$ examined the effect of intramuscular administered of corticosteroids on the labor process using a randomized, controlled study on 66 women with gestational age of 41 weeks and over and favorable cervix (bishop score $>$ or $=7)$. The study group $(\mathrm{n}=32)$ received $10 \mathrm{mg}$ of dexamethasone phosphate intramuscularly in two doses at an interval of 12 hours, and the day after the enrolling administration intravenous oxytocin was given. The control group (n $=33$ ) received only intravenous oxytocin 24 hours after enrolling. The number of patients to enter the active phase of labor was significantly higher in the study group than in the control group $(n=33$ [100\%] vs $\mathrm{n}=29$ [87.9\%], $\mathrm{p}<0.039)$. The mean time interval between induction of labor and the active phase was significantly shorter in the study group than in the control group $(1.7+/-1.5$ hours vs $4+/$ $1.7, \mathrm{P}<0.0001)$, and the mean of oxytocin dose was significantly lower in the study group $(1.15+/-1.5 \mathrm{u}$ vs $4.16+/-2.5 \mathrm{u}, \mathrm{P}<$ $0.0001)$. The study confirmed that induction of labor with the use of intramuscularly injected dexamethasone phosphate reduced the time interval between the induction of labor and the active phase. This is in line with the present study, because the use of dexamethasone enhanced and shortened the second stage of labor.

On the other hand, the present study contradicts with Ogueh1 et al. ${ }^{(6)}$ who explained the effect of dexamethasone on HCG concentrations and suggests that it has a direct inhibitory effect on placental hormone synthesis or secretion. The authors recommended further studies to define the mechanism of action of dexamethasone on placental HCG production.

Furthermore, Kashanian et al. ${ }^{(18)}$ evaluated the effect of dexamethasone administration on labor duration. In this controlled trial on 122 nulliparous women with a full-term pregnancy and a Bishop score of 7 or greater were randomly assigned to receive a single 8-mg dose of dexamethasone or placebo 6 hours before initiation of labor induction. The authors found that the duration of the second stage of labor was also shorter in the dexamethasone group. The effect of dexamethasone on labor duration had many explanations. It has been shown that glucocorticoids induce the synthesis of surfactant protein in human fetal membranes, and suggested that surfactant protein induced prostaglandin E2 synthesis in chorionic trophoblasts - thus playing a role in the initiation of parturition. ${ }^{(20,21)}$ Glucocorticoids are normally circulating stress hormones; however, in the initiation of labor, a stressful situation, their expression is regulated by placentally derived corticotrophin-releasing hormone $(\mathrm{CRH}) .{ }^{(20)}$ Other studies have suggested a role for this hormone on the initiation of active contractions of uterus ${ }^{(22)}$, and also a role for maternal stress hormones. ${ }^{(23)}$

In the present study, there was a statistically significant direct correlation between first stage duration and maternal BMI. This is in accordance with Norman et al. ${ }^{(24)}$ who found that the first stage of labor for obese women is of longer duration and has a slower progression until $6 \mathrm{~cm}$ than the non-obese cohorts, even after adjusting for potential confounding factors. In addition, the current study found a statistically significant correlation between gestational age and first stage duration. This is in harmony with the 
findings of Cheng et al. ${ }^{(25)}$ who reported that women with first stage duration greater than the 95th percentile had significantly higher frequency of gestational ages more than 41 weeks when compared with other groups. Also, our study found a statistically significant inverse correlation between duration of the $1^{\text {st }}$ stage of labor and bishop score. This finds support in the study of Strobel et al. ${ }^{(26)}$ who concluded that Bishop score in a significant predictor of duration of labor. Regarding the association between the second stage duration and the demographic and clinical parameters, the present study found a statistically significant direct correlation between duration of the second stage and maternal age. This is in accordance with Papadias et al. ${ }^{(27)}$ who found that in primigravidas, maternal age was positively correlated with the duration of the second stage of labor. Gomes et al. ${ }^{(28)}$ also revealed that the consistent increase in the average age at pregnancy may exacerbate the main causes of death, raising concerns for the future and prompting the need for emergency facilities nearby maternities.

Canavan et al. ${ }^{(10)}$ made a study on preterm premature rupture of membranes (PPROM) that occur in $3 \%$ of pregnancies and responsible for one third of all preterm births. The study confirmed that treatment using antibiotic and corticosteroid therapy has the strongest evidence for improving neonatal outcome is gestational agedependent and influences local neonatal intensive-care unit (NICU) survival statistics according to the U.S. Preventative Task Force recommendations. ${ }^{(27)}$

Papadias et al. ${ }^{(28)}$ revealed that there is a statistically significant relationship between parity, maternal age at delivery, gestational age, and duration of the second stage of labor. In primigravidas, under the age of 20 years, the second stage of labor was significantly shorter compared to women aged over 40 years, and significantly shorter compared to women between the age of 20 and 40 years. Gestational age at delivery was significantly shorter in women aged over 40 years compared to those under the age of 20 years as well as to those between 20 and 40 years of age. Age was positively correlated to the duration of the second stage of labor and negatively correlated to the gestation age at delivery. In multigravidas, age was negatively correlated to the gestational age at delivery. In primigravidas, maternal age was positively correlated with the duration of the second stage of labor. On the contrary, gestational age at delivery was negatively correlated with maternal age. In multigravidas, a negative correlation between maternal age and gestational age at delivery was statistically significant. Gomes et al. ${ }^{(29)}$ also revealed that the consistent increase in the average age at pregnancy may exacerbate the main causes of death, raising concerns for the future and prompting the need for emergency facilities nearby maternities.

\section{Limitations}

The study had the following limitations: inclusion of a small number of health care facilities, and a small sample size.

\section{Conclusions}

The present study concluded that IM administration of dexamethasone influences the duration of the second stage of labor in primigravidas women, as it resulted in significant shortening of the second stage of labor. This is of great value particularly in primigravidas where 
the long duration of labor is exhausting and troublesome.

\section{Recommendations}

This study provides high-level evidence of direct relevance for clinical practice. Dexamethasone can be used consistently in primigravidas women to enhance the second stage of labor. Further assessments should be conducted to find out the association between other maternal and fetal sociodemographic and clinical data and dexamethasone administration.

\section{References}

1- Kavanagh J, Kelly AJ, Thomas J. Corticosteroids for cervical ripening and induction of labor. Cochrane Database Syst Rev 2006 (2):CD003100.

2- Kavanagh J, Kelly AJ, Thomas J. Corticosteroids for induction of labor. Cochrane Database Syst Rev 2001;(2):CD003100.

3- McAuliffe F, Grimes H, Morrison JJ. Fetal cortisol in relation to labour, intrapartum events and mode of delivery. J Obstet Gynaecol 2004;24(2):129-32.

4- Kalantaridou S, Makrigiannakis A, Zoumakis E, Chrousos GP. Peripheral corticotrophin-releasing hormone is produced in the immune and reproductive systems: actions, potential roles and clinical implications. Front Biosci 2007;12:572-80.

5- Lindsay JR, Nieman LK. The hypothalamic-pituitary-adrenal axis in pregnancy: challenges in disease detection and treatment. Endocr Rev 2005;26(6):775-99.

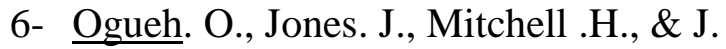
Alaghband-Zadeh. J.A., and Johnson M.R.. Effect of antenatal dexamethasone therapy on maternal plasma human chorionic

gonadotrophin, oestradiol and progesterone. Human Reproduction, 1998 ; 14( 2):303-306.

7- Liu. K., Liu. C.C., and Chia. Y.Y. Effect of dexamethasone on postoperative emesis and pain. Br. J. Anaesth. 1998; 80 (1): 85-86.

8- Liu. k, Hsu. C.C, and Chia.Y.Y. The Effective Dose of Dexamethasone for Antiemesis after Major Gynecological Surgery. the International Anesthesia Research Society, 2013

9- Vidaeff A.C, and Ramin S.M. Antenatal corticosteroids after preterm premature rupture of membranes. $\underline{\text { Clin }}$ Obstet Gynecol. 2011;54(2):337-43.

10- Canavan T.P, Simhan H.N, and Caritis

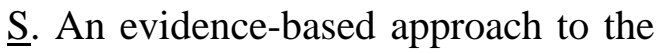
evaluation and treatment of premature rupture of membranes: Part II. Obstet Gynecol Surv. 2004 Sep;59(9):67889.

11-Grgić G, Fatusić Z, and Bogdanović G. [Stimulation of fetal lung maturation with dexamethasone in unexpected premature labor]. Med Arh. 2003;57(5-6):291-4.

12- Pattinson RC. A meta-analysis of the use of corticosteroids in pregnancies complicated by preterm premature rupture of membranes. $\underline{\text { S Afr Med J. }}$ 1999 Aug;89(8):870-3.

13-Movafegh. A, Razazian. M, Hajimaohamadi. F, and Meysamie. A Dexamethasone Added to Lidocaine Prolongs Axillary Brachial Plexus Blockade. Anesthesia and Analgesia the Gold Standard in Anesthesiology IARS. The International Anesthesia Research Society. 2013. Print ISSN: 0003-2999

14-Cummings. C., Napierkowski. D.E., Sanchez.P., Kurz. A., Dalton. E.J., 
Brems. J.J., and Sessler.I.D. Effect of dexamethasone on the duration of interscalene nerve blocks with ropivacaine or bupivacaine. Br. J. Anaesth. 2011; 107 (3): 446-453.

15-Daly, W. M. and Carnwell, R. Nursing roles and levels of practice: a framework for differentiating between elementary, specialist and advancing nursing practice. Journal of Clinical Nursing2003;12: 158-

167.doi: $10.1046 /$ j.13652702.2003.

Polit, D.F., \& Hungler, B.P. Nursing Research Principles and Methods, $6^{\text {th }}$ edition, Lippincott, Philadelphia, Neew York, Baltimore.1999

16- Barkai G, Cohen SB, Kees S, Lusky A, Margalit V, Mashiach S, Schiff E. Induction of labor with use of a Foley catheter and extraamniotic corticosteroids. Am J Obstet Gynecol 1997;177(5): 1145-8.

17- Kashanian M, Dadkhah F, Mokhtari F (b). Effect of intramuscular administration of dexamethasone on the duration of labor. Int $\mathbf{J}$ Gynaecol Obstet. 2008 ;102(3):259-62.

18- Ziaei S, Rosebehani N, Kazeminejad A, Zafarghandi S.The effects of intramuscular administration of corticosteroids on the induction of parturitionJ Perinat Med. 2003;31(2):134-9..

19-Sun K, Brockman D, Campos B, Pitzer B, Myatt L. Induction of surfactant protein A expression by cortisol facilitates prostaglandin synthesis in human chorionic trophoblasts. J Clin Endocrinol Metab 2006;91(12):4988 - 94.

20-Vitoratos N, Papatheodorou DC, Kalantaridou SN, Mastorakos G. "Reproductive" corticotropinreleasing hormone. Ann N Y Acad Sci 2006;1092:310-8.

21-Gitau R, Fisk NM, Glover V. Human fetal and maternal corticotrophin releasing hormone responses to acute stress. Arch Dis Child Fetal Neonatal Ed 2004;89(1):F29-32.

22-Pike. I.L. Maternal stress and fetal responses: evolutionary perspectives on preterm delivery. Am J Hum Biol 2005;17(1): 55-65.

23-Norman SM, Tuuli MG, Odibo AO, Caughey AB, Roehl KA, Cahill AG. The effects of obesity on the first stage of labor. Obstet Gynecol. 2012;120(1):130-5.

24-Cheng YW, Delaney SS, Hopkins LM, Caughey AB. The association between the length of first stage of labor, mode of delivery, and perinatal outcomes in women undergoing induction of labor. Am J Obstet Gynecol. 2009 ;201(5):477.e1-7.

25-Strobel E, Sladkevicius P, Rovas L, De Smet F, Karlsson ED, Valentin L. Bishop score and ultrasound assessment of the cervix for prediction of time to onset of labor and time to delivery in prolonged pregnancy. Ultrasound Obstet Gynecol. 2006;28(3):298-305.

26- Yyonne W. Cheng, Linda M. Hopkins, and Aaron B. Caughey. How long is too long: Does a prolonged second stage of labor in nulliparous women affect maternal and neonatal outcomes? Original Research Article. American Journal of Obstetrics and Gynecology, Volume 191, Issue 3, September 2004;933-938

27-Papadias K, Christopoulos P, Deligeoroglou E, Vitoratos N, Makrakis E, Kaltapanidou P, Tsoukas A, Creatsas G. Maternal age and the duration of the second stage of labor. Ann N Y Acad Sci. 2006;1092:414-7.

28-Gomes M.C, Maria T. Ventura M. T, Nunes R.S How many maternal deaths are there in Portugal? Journal of Maternal-Fetal and Neonatal Medicine. 2012 ;25, (10):1975-1979 


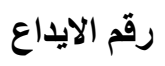

